\title{
On the bialgebra of functional graphs and differential algebras
}

\author{
Maurice Ginocchio
}

Laboratoire de Physique Théorique et Mathématique, Université Paris 7, Tour Centrale -3ème étage, 2, place Jussieu, F-75251 Paris Cedex 05, France

E-Mail: mag@ecr.jussieu.fr

We develop the bialgebraic structure based on the set of functional graphs, which generalize the case of the forests of rooted trees. We use noncommutative polynomials as generating monomials of the functional graphs, and we introduce circular and arborescent brackets in accordance with the decomposition in connected components of the graph of a mapping of $\{1,2, \ldots n\}$ in itself as in the frame of the discrete dynamical systems. We give applications for differential algebras and algebras of differential operators.

Keywords: bialgebraic structure, functional graphs, noncommutative polynomials

\section{Introduction}

We have already described the expansion of $\Delta=\Sigma \lambda^{i} \partial_{i}$, i.e. the powers of a Lie operator in any dimension, in order to find the expression of the flow of formal nonlinear evolution equations [1-3] In the onedimensional case, the explicit expansion can be found first in Comtet [4], and other aspects connected to the ordinary differential equations can be found in Leroux and Viennot [5] ancl Bergeron and Reutenauer [6]. On the other hand, Grossman and Larson [7] introduced several Hopf algebras [8--10] of forests of rooted labeled trees to express the product of finite dimensional vector fields. In this paper we concentrate us on the bialgebra $\mathbf{G}$ of functional graphs, i.e. graphs representing mappings of finite sets in themselves [11-15]. We give only the results without proofs. In a forthcoming paper [16], we develop Hopf algebra structures, computing the antipode and giving detailed aspects and proofs.

In Sect. 1 we consider a bialgebra structure on $\mathbf{G}$ and three interesting subalgebras: $\mathbf{T}$ the set of labeled forests; $\mathbf{S}$ the set of permutation graphs; and $\mathbf{L}$ the set of well labeled forests, i.e. with strictly decreasing labels on the chains toward the roots. Recall that the graded bialgebra $\mathbf{L}$ is sufficient for the calculus of the powers of one derivation [1], and it is extendable in a Hopf algebra, the element of which is known in the computer literature as 'heap ordered trees'. This bialgebra is useful to compute products of derivations or to transform differential monomials in differential algebras [17], and it is interesting to note that the elements $\mathbf{L}_{n}$ ( $n$ edges) can be coded by the words (monomials) of the expansion of $Q_{n} \equiv$ $q_{0}\left(q_{0}+q_{1}\right) \ldots\left(q_{0}+q_{1}+\ldots q_{n-1}\right)$, where $Q^{0} \equiv\left\{q_{0}, q_{1}, \ldots\right\}$ is a noncommutative alphabet. We describe in particular the bialgebra $\mathbf{L}$, first in the polynomial form by the 'factorial' monoid $L^{0}=\left(L_{n}^{0}\right)_{n \geq 0}$, where $L_{n}^{0}$ is the set of words in the expansion of $Q_{n}$, and second, we establish the bijective correspondence between 
$L$ and $\mathbf{L}$. We show that the calculus are easier with $L$, and that the product on $L$ can be expressed in a very natural way. For example, $\left(q_{0}\right)^{n}=Q_{n}$, hence the (exponential) generating function of all the elements of L. We describe principally the formalism in the general case $\mathbf{G}$, and the calculus uses the fields $F_{2}=\{0,1\}$ as well as characteristic zero fields $K$.

In Sect. 2, we describe the link with the graded differential algebra $K\{U\}=\oplus_{r \geq 0} K\{U\}_{r}$ and the graded algebra of differential operators $K\{U, D\}=\oplus_{r \geq 0} K\{U\}_{r} D^{r}$, where $U \equiv\left(u_{1}, u_{2}, \ldots\right)=\left(u_{\beta}\right)_{\beta \geq 1}=$ $\left(u_{\beta}{ }^{\alpha}\right)_{\alpha \geq 0, \beta \geq 1}$ is a set of indeterminates, $D \equiv\left\{\partial_{0}, \partial_{1}, \ldots\right\}$ and the differential indeterminates $u_{\beta}{ }^{\alpha}{ }_{\sigma_{1} \ldots \sigma_{p}}=$ $\partial_{\sigma_{1} \ldots} \ldots \partial_{\sigma_{p}} u_{\beta}{ }^{\alpha}$ generate $K\{U\}_{r}$ [17] This shows that the above Q-calculus, which is a kind of 'dissection' on functional graphs can be used as pre-calculus in differential algebras as well as in discrete dynamical system: [18]

\section{Bialgebra Based on the Semi-group of Functional Graphs}

\subsection{Types of Functional Graphs}

In this paper, a connected functional graph will be called excycle $[13,15]$. In the area of discrete dynamical systems, an excycle is known as a basin of attraction. Consider several graded and filtered sets of labeled functional graphs

(i) $\mathbf{E}$ (resp.G) the set of excycles (resp. functional graphs) and designated by $\mathbf{G}_{n}\left(\operatorname{resp} . \mathbf{G}_{(n)}\right.$ ), the set of functional graphs having (resp. having at most) $n+1$ nodes for $n \geq 0$.

(ii) $\mathbf{R}$ (resp.T) the set of labeled arborescences (resp. forests).

(iii) $\mathbf{C}$ (resp.S) the set of cycles (resp. permutation graphs).

(iv) A (resp.L) the set of well labeled arborescences (resp. forests), i.e. with strictly decreasing labels on the chains toward the root(s).

As in (i), we consider for (ii)-(iv) graduations and filtrations.

\subsection{Free Representation by Q-polynomials}

Let $G_{n}$ be the semigroup of mappings of $\{1,2, \ldots n\}$ in itself ('Semigroup of endofunctions'),

- Card $G_{n}=n^{n}$, and the subsemigroups,

- $T_{n} \equiv\left\{f ; f \in G_{n}, f^{n}=f^{n-1}\right\}$ (i.e. f acyclic and Card $T_{n}=(n+1)^{n-1}$ ),

- $S_{n}$ the symmetric group and Card $S_{n}=n$ !

- $L_{n} \equiv\left\{f ; f \in G_{n}, f(i) \leq i\right\}$ (i.e. $f$ subdiagonal and Card $L_{n}=n$ !).

We have the well known bijections ${ }^{[F-F]}: \mathbf{G}_{n} \approx G_{n}, \mathbf{T}_{n} \approx T_{n}, \mathbf{S}_{n} \approx S_{n}, \mathbf{L}_{n} \approx L_{n}$.

Let $Q \equiv\left\{q_{0}, q_{1}, \ldots\right\}$ be a noncommutative alphabet, $Q^{0} \equiv\left\{q_{0}\right\} \cup Q$ with $q_{0}$ noncommuting with the $q_{i}$ 's, $Q_{n} \equiv\left\{q_{1}, q_{2}, \ldots q_{n}\right\}, Q_{n}^{0} \equiv\left\{q_{0}\right\} \cup Q_{n}$ and $Q^{*}$ (resp. $Q^{0 *}$ ), the corresponding free monoids. Taking $F_{2}=\{0,1\}$ as the field, consider

(i) the $G_{n}$ module $F_{2} Q_{n}$ by the $F_{2}$ linear incidence matrix action of $f \in G_{n}$ as $l_{f} q_{i}=q_{f(i)}$ hence $l_{f} l_{g}=$ $l_{f \bullet g}$. 
(ii) the generating monomial associated with $f$.

By morphism extension, denoted again by $l_{f}$, we define

$$
Q_{f} \equiv q_{f(1)} q_{f(2)} \ldots q_{f(n)}=l_{f} Q_{i_{n}}
$$

where $Q_{\imath_{n}} \equiv q_{1} q_{2} \ldots q_{n}$ is associated with the identity $\iota_{n}$ of $G_{n}$ and $Q \imath_{0}=1$. One again has $l_{f} l_{g}=l_{f} \bullet$.

For the following we consider

(iii) The graded subsets of $Q^{*}$ as $G \equiv\left(G_{n}\right)_{n \geq 0}, T \equiv\left(T_{n}\right)_{n \geq 0}, S \equiv\left(S_{n}\right)_{n \geq 0}, L \equiv\left(L_{n}\right)_{n \geq 0}$, respectively associated with $\mathbf{G}, \mathbf{T}, \mathbf{S}$ and $\mathbf{L}$, with $G_{0}=T_{0}=S_{0}=L_{0}=\{1\}$.

(iv) The corresponding graded $F_{2}$-modules: $F_{2} G, F_{2} T, F_{2} S, F_{2} L$, admit components of degree $n$ which are, respectively, $G_{n}, T_{n}, S_{n}, L_{n}$ modules, with

$$
\operatorname{dim}_{2} G_{n}=n^{n}, \operatorname{dim}_{2} T_{n}=(n+1)^{n-1}, \operatorname{dim}_{2} S_{n}=d i m F_{2} L_{n}=n !
$$

(v) We will denote by $R_{n}$ one of the above subsemi-groups of $G_{n}$ (or of another category).

Similarly, let $R \equiv\left(R_{n}\right)_{n \geq 0},\left(\right.$ resp. $\left.F_{2} R=\oplus_{n \geq 0} F_{2} R_{n}\right)$ be the corresponding graded subsets of $Q^{*}$ (resp. graded $F_{2}$-modules of

$\left.F_{2} G=\oplus_{n \geq 0} F_{2} G_{n}\right)$.

\subsection{Virtual Root and External Product}

Let $f \in G_{n}, I_{0}$ be the set of fixed points of $f$ and $H_{0}$ a subset of $I_{0}$, and set $[p, q] \equiv\{r ; p \leq r \leq q\}$ if $p \leq q$ and $\emptyset$ otherwise.

Define $f^{0}:[1, n] \rightarrow[0, n]$ such that $f^{0}(i)=f(i)$ if $i \notin H_{0}$ and $f^{0}(i)=0$ if $i \in H_{0}$. The ' 0 ' is the label of a virtual root added to the graph representation of $f$, and we will say that $H_{0}$ is 'confined in 0 ', which is a fixed point of $f^{0}$. We call 'extended endofunctions' such functions $f^{0}$, denote by $G_{n}^{0} \equiv[0, n]^{[1, n]}$ their set, and we consider $G_{n}$ as a subset of $G_{n}^{0}$. Similarly, we will have $T_{n}^{0} \supset T_{n}, S_{n}^{0} \supset S_{n}, L_{n}^{0} \supset L_{n}$. Consequently, adding $q_{0}$, we get the extended graded sets $G^{0} \equiv\left(G_{n}^{0}\right)_{n \geq 0}$, the extended graded $F_{2}$-module $F_{2} G^{0}=$ $\oplus_{n \geq 0} F_{2} G_{n}^{0}$ and their substructures $F_{2} T^{0}, F_{2} S^{0}, F_{2} L^{0}$.

Now let $\phi \in G_{m}^{0}, \chi_{0}$ be the characteristic function of $H_{0}=\phi^{-1}(0)$, and write

$$
Q_{\phi}=l_{\phi} Q_{\mathrm{l}_{m}}=q_{\phi(1)} q_{\phi(2)} \ldots q_{\phi(m)}=\prod_{i=1}^{m} q_{\phi(i)}
$$

(cf. Figures 1 and 2).

With $\psi \in G_{n}^{0}$, consider the $F_{2}$-bilinear product in $F_{2} G^{0}$ defined by

$$
Q_{\phi} \cdot Q_{\psi}=Q_{\psi} \prod_{i=1}^{m}\left(q_{\phi(i)+n}+\chi_{0}(i)\left(q_{0}+q_{1}+\ldots q_{n-1}\right)\right)
$$

On the right-hand side we have a sum of concatened monomials, and on the right factor the substitutions $q_{0} \rightarrow q_{0}+q_{1}+\ldots q_{n}$ and $q_{h} \rightarrow q_{h+n}$ when $h \neq 0$. 
On the other hand, the product belongs to $F_{2} G_{m+n}^{0}$. This external product is associated with unit 1 and $F_{2} G^{0}$ is '. graded. To see this consider $i, j, k$ being $\neq 0$, three homogeneous polynomials,

$$
A=A\left(q_{0} ; q_{i}\right) \in F_{2} G_{m}^{0}, B=B\left(q_{0} ; q_{j}\right) \in F_{2} G_{n}^{0}, C=C\left(q_{0} ; q_{k}\right) \in F_{2} G_{p}^{0}
$$

then by (2)

$$
A . B=B\left(q_{0} ; q_{j}\right) A\left(q_{0}+q_{1}+\ldots q_{n} ; q_{i+n}\right)
$$

and so, using deg $(B . C)=n+p$,

$$
\begin{aligned}
\left(A\left(q_{0} ; q_{i}\right) \cdot B\left(q_{0} ; q_{j}\right)\right) \cdot C & =\left(B\left(q_{0} ; q_{j}\right) A\left(q_{0}+q_{1}+\ldots q_{n} ; q_{i+n}\right)\right) \cdot C \\
& =C B\left(q_{0}+q_{1}+\ldots q_{p} ; q_{j+p}\right) A\left(q_{0}+q_{1}+\ldots q_{p}+q_{1+p}+\ldots q_{n+p} ; q_{i+n+p}\right) \\
& =A\left(q_{0} ; q_{i}\right) \cdot\left(C B\left(q_{0}+q_{1}+\ldots q_{p} ; q_{j+p}\right)\right) \\
& \left.=A\left(q_{0} ; q_{i}\right) \cdot\left(B\left(q_{0} ; q_{j}\right)\right) \cdot C\right)
\end{aligned}
$$

Moreover, because $T_{n}, S_{n}, L_{n}$ are subsemi-groups of $G_{n}$, one can see that $F_{2} R^{0}=F_{2} T^{0}, F_{2} S^{0}, F_{2} L^{0}$ are '?' graded subalgebras of $F_{2} G^{0}$. Hence

Proposition 1 Let the sequence $\left(G_{m}^{0}\right)_{m \geq 1}$ of the sets of the extended endofunctions in $\{1,2, \ldots m\}$ and $Q^{0} \equiv\left\{q_{0}, q_{1}, \ldots\right\}$ be a noncommutative alphabet. For $\phi \in G_{m}^{0}$ let $Q_{\phi}=\prod_{i=1}^{m} q_{\phi(i)}$ be the generating monomial of $\phi$ and the graded module $F_{2}$-module $F_{2} G^{0}=\oplus_{n \geq 0} F_{2} G_{n}^{0}$ on $F_{2} \equiv\{0,1\}$ generated by all the $\phi^{\prime} s$.

Then $F_{2} G^{0}$ is a graded algebra for the associative product with unit 1

$$
Q_{\phi} \cdot Q_{\psi}=Q_{\psi} \prod_{i=1}^{m}\left(q_{\phi(i)+n}+\chi_{0}(i)\left(q_{0}+q_{1}+\ldots q_{n-1}\right)\right)
$$

where $\psi \in G_{n}^{0}$ and $\chi_{0}$ is the characteristic function of $\phi^{-1}(0)$.

Moreover, if $\left(R_{m}^{0}\right)_{m \geq 1}$ is a sequence of subsets associated with subsemi-groups of the sequence $\left(G_{m}^{0}\right)_{m \geq 1}$, then $F_{2} R^{0}=\oplus_{n \geq 0} F_{2} R_{n}^{0}$ is a graded subalgebra of $F_{2} G^{0}$.

\subsection{Splitting Operator $\delta_{n} \in F_{2} G^{0}$}

This operator substitutes the $n$-coproduct $\Delta_{n}$ of the Leibniz-Lie type. Associate to $A \in Q^{0 *}$ the left linear operator $\tau_{n} A$ acting on $B \in Q^{0 *}$, such that, if $A \in G_{m}^{0}, B \in G_{n}^{0}$, then $[B]\left(\tau_{n} A\right) \equiv B A$ if $\operatorname{deg} B=n$, and 0 otherwise, where $\mathrm{BA}$ is the concatenation of $\mathrm{B}$ and $\mathrm{A}$.

(i) Now let $f \in G_{m}$ and $H_{0}$ as in Sect. 3, and note first that if

$\tau_{n}$ is viewed as acting on $f$, then for $i \in[1, m]$ one has $\left(\tau_{n} f\right)(i+n)=f(i)+n$, and by $f^{0}(i)=$ $\bar{\chi}_{0}(i) f(i)$ one has $\left(\tau_{n} f^{0}\right)(i+n)=\bar{\chi}_{0}(i)\left(f^{0}(i)+n\right)$, where $\bar{\chi}_{0}=1-\chi_{0}$. According to (2), define for $\phi \in G_{m}^{0}$

$$
\delta_{n} Q_{\phi} \equiv \tau_{n} \prod_{i=1}^{m}\left(q_{\phi(i)+n}+\chi_{0}(i)\left(q_{0}+q_{1}+\ldots q_{n-1}\right)\right)
$$

If $d_{0}=$ Card $H_{0}$ the expansion (4) gives a sum of $(n+1)^{d_{0}}$ generating monomials of functions $\psi_{(\kappa)}$ of $[n+1, n+m]$ into $\{0\} \cup[n+1, n+m]$, and the corresponding functional graphs factorized in commutative excycles. 
The operator $\delta_{n} A$ is left linear on $F_{2} G^{0}$, and (2) can be written

$$
Q_{\phi} \cdot Q_{\psi}=\left[Q_{\psi}\right]\left(\delta_{n} Q_{\phi}\right)
$$

(ii) Moreover, $\delta_{p}$ is a graded antimorphism for '.'

$$
\delta_{p}(A . B)=\left(\delta_{p} B\right)\left(\delta_{p+n} A\right)
$$

where $n=\operatorname{deg} B$ and $p \in \mathbf{N}$.

For this to compute with (5) and $A, B, C$ as in Sect. $3,[C] \delta_{p}(A . B)=(A . B) . C=A .(B . C)=[B . C] \delta_{p+n} A=$ $[C]\left(\delta_{p} B\right)\left(\delta_{p+n} A\right)$. If $p=0$ we recover $A . B=[B] \delta_{n} A$ and $[B] \delta_{k} A=0$ if $k \neq \operatorname{deg} B$.

(iii) Also, $\delta_{n}$ is a power

$$
\delta_{n}=\delta^{n}, \delta=\delta_{1}, \delta^{0}=1
$$

For this to compute, $\delta_{p} \delta_{n} A\left(q_{0} ; q_{i}\right)=\delta_{p} \tau_{n} A\left(q_{0}+q_{1}+\ldots q_{n} ; q_{i+n}\right)=\tau_{n+p} A\left(q_{0}+q_{1}+\ldots q_{p}+q_{1+p}+\right.$ $\left.\ldots q_{n+p} ; q_{i+n+p}\right)=\delta_{n+p} A\left(q_{0} ; q_{i}\right)$.

(iv) Define the left linear operator $\mu$ in $F_{2} G^{0}$ by the expansion

$$
\mu \equiv \sum_{n \geq 0} \delta^{n}
$$

By left linear action of $\mu A$ on $F_{2} G^{0}$, we get $A . B=[B](\mu A)$ for $A, B \in F_{2} G^{0}$ with the antimorphism property

$$
\mu(A . B)=(\mu B)(\mu A)
$$

which express the associativity of '. .

Proposition 2 Let $A \in F_{2} G_{m}^{0}, B \in F_{2} G_{n}^{0}$. Then the splitting linear operator $\delta_{p}$ defined left linearly by $[B] \delta_{p} A \equiv A . B$ if $p=n$, and 0 otherwise, verifies $\delta_{p}=\delta^{p}$ with $\delta=\delta_{1}, \delta^{0}=1$ and $\delta^{p}(A . B)=\left(\delta^{p} B\right)\left(\delta^{p+n} A\right)$. Moreover, $\mu \equiv \sum_{n \geq 0} \delta^{n}$ is an antimorphism in $F_{2} G^{0}$ such that $A . B=[B](\mu A)$.

\subsection{Exponential Generating Function of the Monomials of $L^{0}$}

All the words of $L_{n}^{0}$ (i.e. subdiagonals) are obtained from the expansion of $Q_{n} \equiv q_{0}\left(q_{0}+q_{1}\right) \ldots\left(q_{0}+q_{1}+\right.$ $\left.\ldots q_{n-1}\right) \in F_{2} L^{0}$ and $Q_{0}=1$.

By equation (3), one has $Q_{m} \cdot Q_{n}=Q_{m+n}$, and if $A \in F_{2} L_{m}^{0}, B \in F_{2} L_{n}^{0}$ we have $A . B \in F_{2} L_{m+n}^{0}$, and then we recover that $F_{2} L^{0}$ is stable for the product ' $\therefore$. Because $Q_{1}=q_{0}$, the associativity gives

$$
Q_{n}=\left(q_{0}\right)^{n}
$$

With the $\mathbf{Q}[[\mathrm{t}]]-$-modules on $L^{0}$, one has the exponential generating function

$$
\begin{aligned}
& \exp \left(t q_{0}\right)=\sum_{n \geq 0} \frac{t^{n}}{n !}\left(q_{0}\right)^{n}=\sum_{n \geq 0} \frac{t^{n}}{n !} Q_{n} \\
& \exp \left(s q_{0}\right) \exp \left(t q_{0}\right)=\exp \left((s+t) q_{0}\right)
\end{aligned}
$$




\subsection{Examples}

Consider equations (4) and (5) for $Q_{\psi}=q_{0}^{n}$.

\subsubsection{Rooted Trees with $n=1$}

$\delta\left(q_{0}^{2} q_{1}\right)=\tau\left(q_{0}+q_{1}\right)^{2} q_{2}=\tau q_{0} q_{0} q_{2}+\tau q_{0} q_{1} q_{2}+\tau q_{1} q_{0} q_{2}+\tau q_{1} q_{1} q_{2}$

(Figure 3)

$\left(q_{0}^{2} q_{1}\right) \cdot q_{0}=q_{0}\left(q_{0}+q_{1}\right)^{2} q_{2}=q_{0}^{3} q_{2}+q_{0}^{2} q_{1} q_{2}+q_{0} q_{1} q_{0} q_{2}+q_{0} q_{1} q_{1} q_{2}$

(Figure 4)

\subsubsection{Excycles with $n=2$}

$\delta^{2}\left(q_{3}^{2} q_{1} q_{0}\right)=\tau^{2} q_{5}^{2} q_{3}\left(q_{0}+q_{1}+q_{2}\right)=\tau^{2} q_{5}^{2} q_{3} q_{0}+\tau^{2} q_{5}^{2} q_{3} q_{1}+\tau^{2} q_{5}^{2} q_{3} q_{2}$

(Figure 5)

$q_{3}^{2} q_{1} q_{0} \cdot q_{0}^{2}=q_{0}^{2} q_{5}^{2} q_{3}\left(q_{0}+q_{1}+q_{2}\right)=q_{0}^{2} q_{5}^{2} q_{3} q_{0}+q_{0}^{2} q_{5}^{2} q_{3} q_{1}+q_{0}^{2} q_{5}^{2} q_{3} q_{2}$

(Figure 6)

\section{Differential Algebra}

\subsection{Differential indeterminates}

Let $D \equiv\left\{\partial_{0}, \partial_{1}, \ldots\right\}$ where $\partial_{\alpha} \equiv \partial / \partial \xi^{\alpha}$, the $\alpha^{\text {th }}$ canonical derivation in $\mathbf{S} \equiv K[[\xi]]$ the algebra of formal power series in $\xi \equiv\left\{\xi^{0}, \xi^{1}, \ldots\right\}$, where $K$ is a characteristic zero field. If $\mathbf{S}^{N \times N}$ is the set $U \equiv\left(u_{1}, u_{2}, \ldots\right)=$ $\left(u_{\beta}\right)_{\beta \geq 1}=\left(u_{\beta}^{\alpha}\right)_{\alpha \geq 0, \beta \geq 1}$ with $u_{\beta}^{\alpha} \in \mathbf{S}$, consider $U$ as a set of indeterminates, $u_{\beta}^{\alpha} \sigma_{1} \ldots \sigma_{p}=\partial_{\sigma_{1}} \ldots \partial_{\sigma_{p}} u_{\beta}^{\alpha}$ as differential indeterminates, replace $\mathbf{S}^{N \times N}$ by $K U$, and consider the graded differential algebra $K\{U\}=$ $\oplus_{r \geq 0} K\{U\}_{r}$ and the graded algebra of differential operators $K\{U, D\}=\oplus_{r \geq 0} K\{U\}_{r} D^{r}$.

To each $W \in F_{2} R^{0}$ we associate the differential operator $W(U) \in\{U, D\}$; for example, with $W_{r}(U) \in$ $K\{U\}_{r}$ one has

$$
W(U)=W_{0}(U)+W_{1}(U)^{\alpha} \partial_{\alpha}+W_{2}(U)^{\alpha \beta} \partial_{\alpha} \partial_{\beta}+\ldots=W_{0}(U)+\sum_{r \geq 1}\left\langle W_{r}(U), D^{r}\right\rangle
$$

We will use now the summation convention.

\subsection{Brackets in $K\{U\}$}

Define for $u, v, w \ldots \in U$ the multilinear operations valued in $K\{U\}$.

\subsubsection{Arborescent Brackets (Valued in $K\{U\}_{1}$ )}

$\langle u, v\rangle,\langle u v, w\rangle \ldots,\langle u, v\rangle^{\beta} \equiv u^{\alpha} v^{\beta}{ }_{\alpha}$, hence $\langle\langle u, v\rangle, D\rangle=u^{\alpha} v^{\beta}{ }_{\alpha} \partial_{\beta}$ (1 fixed point sent to ' 0 ') $\langle u v, w\rangle^{\gamma} \equiv$ $u^{\alpha} v^{\beta} w_{\alpha \beta}^{\gamma}$, hence

$$
\langle\langle u, v\rangle, D\rangle=u^{\alpha} v^{\beta} w_{\alpha \beta}^{\gamma} \partial_{\gamma}
$$

Also, for $A \in K\{U\}_{r}, B \in K\{U\}_{s}, \quad\langle A, B\rangle^{\beta_{1} \ldots \beta_{s}} \equiv A^{\alpha_{1} \ldots \alpha_{r}} B^{\beta_{1} \ldots \beta_{s}} \alpha_{1} \ldots \alpha_{r}$.

\subsubsection{Circular Brackets (Valued in $K\{U\}_{0}$ )}

$(u) \equiv u_{\alpha}^{\alpha} \quad$ (1 fixed point), $(u)(v) \equiv u_{\alpha}^{\alpha} \nu_{\beta}^{\beta} \quad$ (2 fixed points)

$$
(u, v) \equiv u_{\beta}^{\alpha} v_{\alpha}^{\beta}(2-c y c l e),(u, v, w) \equiv u_{\gamma}^{\alpha} v^{\beta}{ }_{\alpha} w^{\gamma}{ }_{\beta}(3-c y c l e)
$$




\subsubsection{Mixed Brackets (Valued in $K\{U\}_{0}$ )}

Let $E$ be a proper excycle (i.e. with no fixed point); we can write it $E=\left(A_{i_{1}}, A_{i_{2}}, \ldots A_{i_{p}}\right)$, where the $A_{i_{k}}$ 's are arborescences with root $i_{k}$. If in each arborescence $A_{i_{k}}$ is reduced to its root $i_{k}$, we recover simply a cycle $E=\left(i_{1}, i_{2}, \ldots i_{p}\right)$.

Now let $F_{k}$ be the forest under $i_{k}$, i.e. obtained by cutting the root of $A_{i_{k}}$, and defined with $F_{i_{k}}(U) \equiv$ $F_{i_{k}}\left(\left\{u_{j}\right\} ; j \in N_{i_{k}}\right)$, where $N_{i_{k}}$ is the set of nodes of $F_{i_{k}}$ :

$$
\left.E(U) \equiv\left(u_{i_{1}}^{\left\langle F_{i_{1}}\right\rangle}, u_{i_{2}}{ }_{i_{i_{2}}}\right\rangle, \ldots u_{i_{p}}^{\left\langle F_{i_{p}}\right\rangle}\right) \equiv\left\langle F_{i_{1}}(U), u_{i_{1}}{ }^{\alpha_{1}}{ }^{\alpha_{p}}\right\rangle\left\langle F_{i_{2}}(U), u_{i_{2}}{ }^{\alpha_{2}} \alpha_{1}\right\rangle \ldots\left\langle F_{i_{p}}(U), u_{i_{p}}{ }^{\alpha_{p}} \alpha_{p-1}\right\rangle
$$

\subsection{Action of $F_{2} R^{0}$}

Moreover, $F_{2} R^{0}$ operates $K$-linearly in $K\{U\}$ with values in $K\{U, D\}$. For this let $\phi \in G_{m}^{0}, H_{0}=\phi^{-1}(0)$ for $j \in[0, m], I=[1, m]$, and $H=u_{\beta_{1}} u_{\beta_{2}} \ldots u_{\beta_{m}} \in U^{*}$, a word on $U$ of length $m$. Then the action is

$$
Q_{\phi}=\prod_{i \in I} q_{\phi(i)} \rightarrow Q_{\phi}(H)=\prod_{j \in I}\left(\prod_{i \in H_{j}} \partial_{\alpha_{i}}\right) u^{\alpha_{j}}{ }_{\beta_{j}} \prod_{k \in H_{0}} \partial_{\alpha_{k}}
$$

The differential monomial $Q_{\phi}(H)$ is such that $u_{\beta_{j}}$ is associated with $j$ in the domain $I$ of $\phi$. If $d_{j}$ is the degree in $q_{j}$ (in-degree of the node labeled by ' ${ }^{\prime}$ '), then $u^{\alpha_{j}} \beta_{j}$ is derived $d_{j}$ times and the indices of derivation are related to the places of the $q_{j}$ 's in the word. Similarly, the differential operator $D^{r}$ is characterized by the number $r$ (degree of the root) of the $q_{0}$ 's and their places. So we can summarize: In a word $A \in R^{0}$ where $q_{j}$ is at the place $(i)$, then in $A(H)$ the $j^{\text {th }}$ letter of $H$ is derived according to $i$, i.e. $\partial_{\alpha_{i}}$ acts. One has, in particular, taking $H=u_{1} u_{2}, \ldots$ :

$$
\begin{aligned}
& \text { Arborescent brackets } \\
& 1(U)=1 \\
& q_{0}(U)=u_{1}{ }^{\alpha_{1}} \partial_{\alpha_{1}}=\left\langle u_{1}, D\right\rangle \\
& q_{0} q_{0}(U)=u_{1} \alpha_{1} u_{2}{ }^{\alpha_{2}} \partial_{\alpha_{1}} \partial_{\alpha_{2}}=\left\langle u_{1} u_{2}, D^{2}\right\rangle \\
& q_{0} q_{1}(U)=u_{1}{ }^{\alpha_{1}} \alpha_{2} u_{2}{ }^{\alpha_{2}} \partial_{\alpha_{1}}=\left\langle\left\langle u_{2}, u_{1}\right\rangle, D\right\rangle \\
& q_{3} q_{3} q_{0}(U)=u_{1} \alpha_{1} u_{2}{ }^{\alpha_{2}} u_{3}{ }^{\alpha_{3}} \alpha_{1} \alpha_{2} \partial_{\alpha_{3}}=\left\langle\left\langle u_{1} u_{2}, u_{3}\right\rangle, D\right\rangle \\
& q_{0} q_{0} q_{2} q_{2}(U)=u_{1}{ }^{\alpha_{1}} u_{2}{ }^{\alpha_{2}} \alpha_{3} \alpha_{4} u_{3}^{\alpha_{3}} u_{4}^{\alpha_{4}} \partial_{\alpha_{1}} \partial_{\alpha_{2}}=\left\langle u_{1}\left\langle u_{3} u_{4}, u_{2}\right\rangle, D^{2}\right\rangle \\
& \text { Circular brackets } \\
& q_{1}(U)=u_{1}{ }^{\alpha_{1}} \alpha_{1}=\left(u_{1}\right) \\
& q_{1} q_{2}(U)=u_{1} \alpha_{1} \alpha_{1} u_{2}{ }^{\alpha_{2}} \alpha_{2}=\left(u_{1}\right)\left(u_{2}\right) \\
& q_{2} q_{1}(U)=u_{1}{ }^{\alpha_{1}} \alpha_{2} u_{2}{ }^{\alpha_{2}} \alpha_{1}=\left(u_{1}, u_{2}\right) \\
& q_{3} q_{1} q_{2}(U)=u_{1} \alpha_{1} \alpha_{3} u_{2}{ }^{\alpha_{2}} \alpha_{1} u_{3}{ }^{\alpha_{3}} \alpha_{2}=\left(u_{1}, u_{2}, u_{3}\right)
\end{aligned}
$$

\subsection{Product of Differential Operators}

The product (2) on words with correspondence (15) gives the product of differential operators. We state, without proof,

Proposition 3 Let the graded differential algebra $K\{U\}=\oplus_{r \geq 0} K\{U\}_{r}$ and the graded algebra of differential operators $K\{U, D\}=\oplus_{r \geq 0} K\{U\}_{r} D^{r}$. Let $\phi \in G_{m}^{0}, I=[1, m], H_{j}=\phi^{-1}(j)$ for $j \in[0, m]$ and $H=$ $u_{\beta_{1}} u_{\beta_{2}} \ldots u_{\beta_{m}}$ a word on $U$ of length $m$. Then the mapping of $F_{2} G^{0}$ into $K\{U, D\}$ which associates to the generating monomial $Q_{\phi}=\prod_{i \in I} q_{\phi(i)}$ of $\phi$ the differential operator $Q_{\phi}(H)=\prod_{j \in I}\left(\prod_{i \in H_{j}} \partial_{\alpha_{i}}\right) u^{\alpha_{j}} \beta_{j} \prod_{k \in H_{0}} \partial_{\alpha_{k}}$ 
is a morphism, such that if $\psi \in G_{n}^{0}$ and $K$ is a word on $U$ of lengthn, one has $Q_{\phi}(H) Q_{\psi}(K)=\left(Q_{\phi} \cdot Q_{\psi}\right)(K H)$, where $K H$ is the concatenation of $K$ and $H$.

Example

$$
\begin{gathered}
A=q_{0}, B=q_{2} q_{1} q_{0}, H=u_{4}, K=u_{1} u_{2} u_{3} \\
A \cdot B=q_{2} q_{1} q_{0}\left(q_{0}+q_{1}+q_{2}+q_{3}\right)=q_{2} q_{1} q_{0} q_{0}+q_{2} q_{1} q_{0} q_{1}+q_{2} q_{1} q_{0} q_{2}+q_{2} q_{1} q_{0} q_{3}
\end{gathered}
$$

(Figure 7)

$$
\begin{gathered}
A(H)=\left\langle u_{1}, D\right\rangle, B(K)=\left(u_{1}, u_{2}\right)\left\langle u_{3}, D\right\rangle, \\
A(H) B(K)=\left(u_{1}, u_{2}\right)\left\langle u_{3} u_{4}, D^{2}\right\rangle+\left\langle u_{4},\left(u_{1}, u_{2}\right)\right\rangle\left\langle u_{3}, D\right\rangle+\left(u_{1}, u_{2}\right)\left\langle\left\langle u_{4}, u_{3}\right\rangle, D\right\rangle
\end{gathered}
$$

Observe that:

$$
\left\langle u_{4},\left(u_{1}, u_{2}\right)\right\rangle\left\langle u_{3}, D\right\rangle=u_{1}{ }^{\alpha_{1}} \alpha_{2} \alpha_{4} u_{2}{ }^{\alpha_{2}} \alpha_{1} u_{3}{ }^{\alpha_{3}} u_{4}{ }^{\alpha_{4}} \partial_{\alpha_{3}}+u_{1}{ }^{\alpha_{1}}{ }_{\alpha_{2}} u_{2}{ }^{\alpha_{2}} \alpha_{1} \alpha_{4} u_{3}{ }^{\alpha_{3}} u_{4}{ }^{\alpha_{4}} \partial_{\alpha_{3}}
$$

which corresponds to $q_{2} q_{1} q_{0}\left(q_{1}+q_{2}\right)$, i.e. the second and third terms in the graph expansion.

\section{Appendix}

To view Figures 1-7, click here. To return to the main paper, click on the red box.

\section{References}

[1] Ginocchio, M. (1995). Universal expansion of the powers of a derivation, Letters in Math. Phys. 34(4), 343-364.

[2] Ginocchio, M. and Irac-Astaud, M. (1985). A recursive linearization process for evolution equations. Reports on Math. Phys. 21, 245-265.

[3] Steeb, W. H. and Euler, N. (1988). Nonlinear Evolution Equations and Painlevé Test. World Scientific.

[4] Comtet, L. (1973). Une formule explicite pour les puissances successives de l'opérateur de dérivation de Lie. Comm. Roy. Acad. Sci. 276 A, 165-168.

[5] Leroux, P. and Viennot, G. (1986). Combinatorial resolution of systems of differential equations I: ordinary differential equations. Actes du colloque de combinatoire énumérative, Montréal. Lecture Notes in Mathematics 1234, pp. 210-245. Springer-Verlag.

[6] Bergeron, F. and Reutenauer, C. (1987). Une interprétation combinatoire des puissances d'un opérateur différentiel linéaire. Ann. Sci. Math. Quebec 11, 269-278.

[7] Grossman, R. and Larson, R. G. (1989). Hopf-algebraic structures of families of trees. J. Algebra 126, $184-210$.

[8] Joni, A. A. and Rota, G. C. (1979). Coalgebras and bialgebras in combinatorics. Studies. in Appl. Math. 61, 93-139. 
[9] Nichols, W. and Sweedler, M. E. (1980). Hopf algebras and combinatorics, in 'Umbral calculus and Hopf algebras'. Contemp. Math. 6.

[10] Sweedler, M. E. (1969). Hopf Algebras. Benjamin.

[11] Berge, C. (1983). Graphes. Gauthier-Villars.

[12] Comtet, L. (1974). Advanced Combinatorics. Reidel.

[13] Denes, J. (1968). On transformations, transformation-semigroups and graphs. In Erdös-Katona, editor, Theory of Graphs. Academic Press, pp. 65-75.

[14] Foata, D. and Fuchs, A. (1970). Réarrangements de fonctions et dénombrements. J. Comb. Theory 8, 361-375.

[15] Harary, F. (1959). The number of functional digraphs. Math. Annalen 138, 203-210.

[16] Ginocchio, M. On the Hopf algebra of functional graphs and differential algebras. Discr. Math. To appear.

[17] Kaplansky, I. (1976). Introduction to Differential Algebras. Springer-Verlag.

[18] Robert, F. (1995). Les systèmes Dynamiques Discrets. Springer-Verlag. 